\title{
Three Natural Generalizations of Fedosov Quantization ${ }^{\star}$
}

Klaus BERING

Institute for Theoretical Physics \& Astrophysics, Masaryk University, Kotlárská 2, CZ-611 37 Brno, Czech Republic

E-mail: bering@physics.muni.cz

Received May 19, 2008, in final form February 14, 2009; Published online March 25, 2009 doi:10.3842/SIGMA.2009.036

\begin{abstract}
Fedosov's simple geometrical construction for deformation quantization of symplectic manifolds is generalized in three ways without introducing new variables: (1) The base manifold is allowed to be a supermanifold. (2) The star product does not have to be of Weyl/symmetric or Wick/normal type. (3) The initial geometric structures are allowed to depend on Planck's constant.
\end{abstract}

Key words: deformation quantization; Fedosov quantization; star product; supermanifolds; symplectic geometry

2000 Mathematics Subject Classification: 53D05; 53D55; 58A15; 58A50; 58C50; 58Z05

\section{Introduction}

The aim of this paper is to give natural generalizations of Fedosov's simple geometrical construction for deformation quantization on a symplectic manifold $M$ [21, 22, 23, 24]. In short, the term "deformation quantization" refers to the construction of an associative $*$ product that is an expansion in some formal parameter $\hbar$, and whose leading behavior is controlled by a geometric structure $m^{i j}$, which usually (but not always) is a symplectic structure [8]. Fedosov quantization, in its most basic form, is a deformation quantization recipe that relies on yet another geometric input in form of a compatible torsion-free tangent bundle connection $\nabla$. It is an important feature of Fedosov's $*$ product that it is a global construction, which applies to a manifold as a whole, and not just a local neighborhood or vector space. Another characteristic feature that sets Fedosov's approach apart from most other methods is the assemblage of a globally welldefined, flat connection $D$. We discuss in this paper the following three natural generalizations of Fedosov's original construction:

1. We let the base manifold be an arbitrary smooth supermanifold. Previous works on superizing Fedosov's construction use Batchelor's theorem (which in turn relies on the existence of a partition of unity) to give a non-canonical identification of the supermanifold with the ringed space of sections of the exterior algebra of a vector bundle, so that the bosonic and fermionic variables are associated with base and fiber directions in this vector bundle, respectively, see e.g., $[13,44]$. On the contrary, we will treat bosons and fermions on equal footing, and will locally allow the use of arbitrary coordinate systems. This of course requires that we pay special attention to sign factors, but it will be more elementary and more general - in the sense that we will not need any major results from the theory of supermanifolds.

${ }^{\star}$ This paper is a contribution to the Special Issue on Deformation Quantization. The full collection is available at http://www.emis.de/journals/SIGMA/Deformation_Quantization.html 
2. We do not assume that $m^{i j}$ is antisymmetric. This is sometimes referred to as the $*$ product does not have to be of Weyl/symmetric type. It is also not necessarily of the Wick/normal type [9, 10, 11, 36, 14, 18, 42, 19].

3. We let the two geometric input data $m^{i j}$ and $\nabla$ depend on Planck's constant $\hbar$. A priori, such a generalization is a non-trivial matter, since Planck's constant $\hbar$ is charged with respect to the pertinent resolution degree, cf. equation (6.1). Moreover, for $\hbar$-dependent structures, there is no analogue of Darboux' theorem at our disposal, cf. discussion below equation (3.7).

Certain aspects of each of the three generalizations have appeared in the literature, but never simultaneously. Our main point is that the three generalizations taken together constitute a natural habitat for Fedosov's original variables $x^{i}, c^{i} \equiv d x^{i}, y^{i}$ and $\hbar$, without destroying the simplicity of his construction.

The paper starts with listing the basic setup and assumptions in Sections 2-3. Then follows an introduction of the relevant tools: An algebra $\mathcal{A}$ of covariant tensors in Sections $4-5$, the Fedosov resolution degree in Section 6, the $\circ$ product in Sections 7-9, the Koszul-Tate differential $\delta$ and its cohomology in Sections 10-11. Thereafter is given a discussion of Riemann curvature tensors on supermanifolds in Sections 12-13. The flat $D$ connection, whose existence is guaranteed by Fedosov's 1st theorem (Theorem 1), is discussed in Sections 14-16. Fedosov's 2nd theorem (Theorem 2), which establishes an algebra isomorphism between symbols and horizontal zeroforms, is discussed in Section 17. Finally, the * product is constructed in the last Section 18.

We shall focus on the existence of the construction and skip the important question of uniqueness/ambiguity/equivalence/classification of $*$ products for brevity. In detail, this paper is an elaboration of the material covered on p. 138-147 in Fedosov's book [24] subjected to the above generalizations 1-3. In particular, what we refer to as Fedosov's 1st and 2nd theorems (Theorems 1 and 2) are generalizations of Theorems 5.2.2 and 5.2.4 in [24], respectively. Some other references that deal with Fedosov's original construction are [43, 31, 40, 26, 45, 30]. Historically, De Wilde and Lecomte were the first to prove the existence of an associative $*$ product on every symplectic manifold [46]. The same question for Poisson manifolds (which may be degenerate) was proved by Kontsevich using ideas from string theory [39]. Cattaneo et al. gave an explicit construction in the Poisson case that merges Kontsevich's local formula with Fedosov's flat $D$ connection approach $[15,16]$.

General remarks about notation. We shall work with smooth finite-dimensional supermanifolds in terms of charts and atlases (as opposed to, e.g., ringed spaces or functors of points). We shall sometimes make use of local formulas, because these often provide the most transparent definitions of sign conventions on supermanifolds. We stress that all formulas in this paper, if not written in manifestly invariant form, hold with respect to any coordinate system, and they transform covariantly under general coordinate transformations. Adjectives from supermathematics such as "graded", "super", etc., are implicitly implied. We will also follow commonly accepted superconventions, such as, Grassmann-parity $\varepsilon$ is only defined modulo 2, and "nilpotent" means "nilpotent of order 2". The sign conventions are such that two exterior forms $\xi$ and $\eta$, of Grassmann-parity $\varepsilon_{\xi}, \varepsilon_{\eta}$ and exterior form degree $p_{\xi}, p_{\eta}$, respectively, commute in the following graded sense

$$
\eta \wedge \xi=(-1)^{\varepsilon_{\xi} \varepsilon_{\eta}+p_{\xi} p_{\eta}} \xi \wedge \eta
$$

inside the exterior algebra. We will often not write exterior wedges " $\wedge$ " nor tensor multiplications " $\otimes$ " explicitly. Covariant and exterior derivatives will always be from the left, while partial derivatives can be from either left or right. We shall sometimes use round parenthesis "( )" to indicate how far derivatives act, see e.g., equations (2.1) and (11.1). 


\section{Basic settings and assumptions}

Consider a manifold $M$ with local coordinates $x^{i}$ of Grassmann-parity $\varepsilon_{i} \equiv \varepsilon\left(x^{i}\right)$. The classical limit

$$
f * g=f g+\frac{i \hbar}{2}\left(f \frac{\overleftarrow{\partial^{r}}}{\partial x^{j}}\right) m^{j k}\left(\frac{\overrightarrow{\partial^{\ell}}}{\partial x^{k}} g\right)+\mathcal{O}\left(\hbar^{2}\right), \quad f, g \in C^{\infty}(M)[[\hbar]] \equiv \mathcal{A}_{00}
$$

of the sought-for associative $*$ multiplication is prescribed by a Grassmann-even contravariant $(2,0)$ tensor

$$
m=m^{i j} \partial_{j}^{\ell} \otimes \partial_{i}^{\ell}, \quad \varepsilon(m)=0, \quad \varepsilon\left(\partial_{i}^{\ell}\right)=\varepsilon_{i} .
$$

(The letter " $m$ " is a mnemonic for the word "multiplication".) The tensor

$$
m^{i j}=m^{i j}(x ; \hbar)
$$

can in principle be a formal power series in Planck's constant $\hbar$. (The quantum corrections to $m^{i j}$ do not enter actively into the classical boundary condition (2.1), but they will nevertheless affect the Fedosov implementation of the $*$ multiplication at higher orders in $\hbar$, as we shall see in equation (7.1) below.) Usually one demands [8] that the classical unit function $\mathbf{1} \in C^{\infty}(M)$ serves as a unit for the full quantum algebra $\left(\mathcal{A}_{00},+, *\right)$ :

$$
f * \mathbf{1}=\mathbf{1} * f=f, \quad f \in C^{\infty}(M)[[\hbar]] \equiv \mathcal{A}_{00} .
$$

(The notation $\mathcal{A}_{00}$ will be explained in Section 4.) Let $m^{T}$ denote the transposed tensor,

$$
\left(m^{T}\right)^{i j}:=(-1)^{\varepsilon_{i} \varepsilon_{j}} m^{j i} .
$$

It will be necessary to assume that the antisymmetric part

$$
\omega^{i j}:=\frac{1}{2}\left(m^{i j}-\left(m^{T}\right)^{i j}\right)=-(-1)^{\varepsilon_{i} \varepsilon_{j}} \omega^{j i}
$$

of the tensor $m^{i j}$ is non-degenerate, i.e., that there exists an inverse matrix $\omega_{i j}$ such that

$$
\omega_{i j} \omega^{j k}=\delta_{i}^{k}
$$

(Note that this does not necessarily imply that $m^{i j}$ itself has to be non-degenerate.) Next, let there be given a torsion-free connection $\nabla: \Gamma(T M) \times \Gamma(T M)[[\hbar]] \rightarrow \Gamma(T M)[[\hbar]]$ that preserves the $m$-tensor

$$
(\nabla m)=0 .
$$

In local coordinates, the covariant derivative $\nabla_{X}$ along a vector field $X=X^{i} \partial_{i}^{\ell}$ is given as [12]

$$
\nabla_{X}=X^{i} \nabla_{i}, \quad \nabla_{i}=\frac{\overrightarrow{\partial^{\ell}}}{\partial x^{i}}+\partial_{k}^{r} \Gamma_{i j}^{k} \overrightarrow{d x^{j}}
$$

Equivalently, $\nabla=d+\Gamma=d x^{i} \otimes \nabla_{i}: \Gamma(T M) \rightarrow \Gamma\left(T^{*} M \otimes T M\right)$, where

$$
\Gamma=d x^{i} \otimes \partial_{k}^{r} \Gamma_{i j}^{k} \overrightarrow{d x}^{j}
$$


Here $\partial_{i}^{r} \equiv(-1)^{\varepsilon_{i}} \partial_{i}^{\ell}$ are not usual partial derivatives. In particular, they do not act on the Christoffel symbols $\Gamma_{i j}^{k}$ in equations (2.5) and (2.6). Rather they are a dual basis to the one-forms $\overrightarrow{d x^{i}}$ :

$$
\overrightarrow{d x^{i}}\left(\partial_{j}^{r}\right)=\delta_{j}^{i} .
$$

Phrased differently, the $\partial_{i}^{r}$ are merely bookkeeping devices, that transform as right partial derivatives under general coordinate transformations. (To be able to distinguish them from true partial derivatives, the differentiation variable $x^{i}$ on a true partial derivative $\partial / \partial x^{i}$ is written explicitly.) The assumption (2.4) reads in local coordinates

$$
0=\left(\nabla_{i} m^{j k}\right)=\left(\frac{\overrightarrow{\partial^{\ell}}}{\partial x^{i}} m^{j k}\right)+\Gamma_{i}{ }_{n} m^{n k}+(-1)^{\varepsilon_{j}\left(\varepsilon_{k}+\varepsilon_{n}\right)} \Gamma_{i}{ }_{n}{ }_{n} m^{j n},
$$

where we have introduced a reordered Christoffel symbol

$$
\Gamma_{i}{ }^{k}{ }_{j}:=(-1)^{\varepsilon_{i} \varepsilon_{k}} \Gamma^{k}{ }_{i j} .
$$

Note that the connection $\nabla$ will also preserve the transposed tensor $m^{T}$, and therefore, by linearity, the antisymmetric part $\omega^{i j}$. We shall later explain why it is crucial that the connection $\nabla$ is torsion-free, see comment after equation (16.1). The Christoffel symbols $\Gamma_{i j}^{k}=\Gamma_{i j}^{k}(x ; \hbar)$ for the connection $\nabla$ is allowed to be a formal power series in Planck's constant $\hbar$. Finally, we mention that one traditionally imposes a reality/hermiticity condition on the connection $\nabla$, the multiplicative structure $m^{i j}$ and the $*$ product. However, we shall for simplicity skip the reality/hermiticity condition in this paper.

\section{The two-form $\omega$ is symplectic}

The inverse matrix $\omega_{i j}$ with lower indices has the following graded skewsymmetry

$$
\omega_{i j}=(-1)^{\left(\varepsilon_{i}+1\right)\left(\varepsilon_{j}+1\right)} \omega_{j i},
$$

cf. equation (2.3). That equation (3.1) should be counted as an skewsymmetry (as opposed to a symmetry) is perhaps easiest to see by defining the slightly modified matrix

$$
\tilde{\omega}_{i j}:=\omega_{i j}(-1)^{\varepsilon_{j}} .
$$

Note that the two matrices $\omega_{i j}$ and $\tilde{\omega}_{i j}$ are identical for bosonic manifolds. Then the equation (3.1) translates into the more familiar type of graded antisymmetry,

$$
\tilde{\omega}_{i j}=-(-1)^{\varepsilon_{i} \varepsilon_{j}} \tilde{\omega}_{j i} .
$$

The skewsymmetry means that the inverse matrix can be viewed as a two-form

$$
\omega:=\frac{1}{2} c^{i} \omega_{i j} c^{j}=-\frac{1}{2} c^{j} c^{i} \tilde{\omega}_{i j} \in \Gamma\left(\bigwedge^{2}\left(T^{*} M\right)\right)[[\hbar]] \equiv \mathcal{A}_{20} .
$$

Here

$$
c^{i} \equiv d x^{i}
$$

is the usual basis of one-forms, and

$$
d:=c^{i} \frac{\overrightarrow{\partial^{\ell}}}{\partial x^{i}}
$$


denotes the de Rham exterior derivative on $M$. It follows from assumption (2.4) that the connection $\nabla$ preserves the two-form

$$
0=\left(\nabla_{i} \tilde{\omega}_{j k}\right)=\left(\frac{\overrightarrow{\partial^{\ell}}}{\partial x^{i}} \tilde{\omega}_{j k}\right)-\left((-1)^{\varepsilon_{i} \varepsilon_{j}} \Gamma_{j, i k}-(-1)^{\varepsilon_{j} \varepsilon_{k}}(j \leftrightarrow k)\right),
$$

where the lowered Christoffel symbol $\Gamma_{k, i j}$ is defined as

$$
\Gamma_{k, i j}:=\omega_{k n} \Gamma^{n}{ }_{i j}(-1)^{\varepsilon_{j}} .
$$

The two-form $\omega$ is closed

$$
(d \omega)=0,
$$

or equivalently,

$$
\sum_{\text {cycl. } i, j, k}(-1)^{\varepsilon_{i} \varepsilon_{k}}\left(\frac{\overrightarrow{\partial^{\ell}}}{\partial x^{i}} \tilde{\omega}_{j k}\right)=0 .
$$

The closeness relation (3.5) is not an extra assumption. It follows from equation (3.4), because the connection $\nabla$ is torsion-free,

$$
T^{k}{ }_{i j}:=\Gamma^{k}{ }_{i j}+(-1)^{\left(\varepsilon_{i}+1\right)\left(\varepsilon_{j}+1\right)}(i \leftrightarrow j)=0,
$$

or equivalently, in terms of the lowered Christoffel symbol,

$$
\Gamma_{k, i j}=(-1)^{\varepsilon_{i} \varepsilon_{j}}(i \leftrightarrow j) .
$$

It is practical to call a non-degenerate closed two-form $\omega_{i j}$ a symplectic structure, even if it depends on Planck's constant $\hbar$. The inverse structure, i.e., the corresponding Poisson structure $\omega^{i j}$ satisfies the Jacobi identity

$$
\sum_{\text {cycl. } i, j, k}(-1)^{\varepsilon_{i} \varepsilon_{k}} \omega^{i n}\left(\frac{\overrightarrow{\partial^{\ell}}}{\partial x^{n}} \omega^{j k}\right)=0
$$

Note that we cannot rely on Darboux' theorem, i.e., we will not be guaranteed a cover of Darboux coordinate patches in which the $\omega^{i j}$ is constant. The issue is that, on one hand, the symplectic structure is allowed to depend on Planck's constant $\hbar$, but, on the other hand, we shall not allow coordinate transformations $x^{i} \rightarrow x^{\prime j}=x^{\prime j}(x)$ that depend on $\hbar$. Luckily, as we shall see, Darboux patches play no rôle in the Fedosov construction. In fact, as we have mentioned before, all formulas in this paper, if not written in manifestly invariant form, hold with respect to any coordinate system, and they transform covariantly under general coordinate transformations.

The classical Poisson bracket is given by the famous quantum correspondence principle [17]

$$
\{f, g\}_{\mathrm{cl}}:=\left(f \frac{\overleftarrow{\partial^{r}}}{\partial x^{j}}\right) \omega_{(0)}^{j k}\left(\frac{\overrightarrow{\partial^{\ell}}}{\partial x^{k}} g\right)=\lim _{\hbar \rightarrow 0} \frac{1}{i \hbar}[f *, g], \quad f, g \in C^{\infty}(M)
$$

Here

$$
[f *, g]:=f * g-(-1)^{\varepsilon_{f} \varepsilon_{g}} g * f=i \hbar\{f, g\}_{\mathrm{cl}}+\mathcal{O}\left(\hbar^{2}\right), \quad f, g \in C^{\infty}(M)[[\hbar]] \equiv \mathcal{A}_{00},
$$

is the $*$ commutator, and

$$
\omega_{(0)}^{i j}:=\lim _{\hbar \rightarrow 0} \omega^{i j} .
$$


It is easy to show that every symplectic manifold $(M ; \omega)$ has a torsion-free $\omega$-preserving connection $\nabla$, see Section 2.5 in [24] for the bosonic case. However, it is not true that every manifold $(M ; m)$ with a multiplicative structure $m^{i j}$ supports a torsion-free $m$-preserving connection $\nabla$, cf. assumption equation (2.4). The symmetric part

$$
g^{i j}:=\frac{1}{2}\left(m^{i j}+\left(m^{T}\right)^{i j}\right)=(-1)^{\varepsilon_{i} \varepsilon_{j}} g^{j i}
$$

of the tensor $m^{i j}$ needs to be compatible with the symplectic structure $\omega$ in a certain sense. In the special case where $g^{i j}=0$, we return to the usual Fedosov quantization $m^{i j}=\omega^{i j}$, which corresponds to a Weyl/symmetric type $*$ product. In the generic case where $g^{i j}$ has full rank, there will exist an inverse matrix $g_{i j}$, which constitute a (pseudo) Riemannian metric, and there will hence exist a corresponding unique Levi-Civita connection $\nabla^{L C}$. In this non-degenerate case, the necessary and sufficient conditions are $\nabla=\nabla^{L C}$ and $\left(\nabla_{i}^{L C} \omega^{j k}\right)=0$. This is for instance satisfied for (pseudo) Kähler manifolds $(M ; \omega ; g)$, cf. [9, 10, 11, 36, 14, 18, 42, 19].

\section{Covariant tensors}

Let

$$
\mathcal{A}_{m n}:=\Gamma\left(\bigwedge^{m}\left(T^{*} M\right) \otimes \bigvee^{n}\left(T^{*} M\right)\right)[[\hbar]]
$$

be the vector space of $(0, m+n)$-tensors $a_{i_{1} \ldots i_{m} j_{1} \ldots j_{n}}(x ; \hbar)$ that are antisymmetric with respect to the first $m$ indices $i_{1} \ldots i_{m}$ and symmetric with respect to the last $n$ indices $j_{1} \ldots j_{n}$. Phrased differently, they are $m$-form valued symmetric $(0, n)$-tensors. As usual, it is practical to introduce a coordinate-free notation

$$
a_{m n}(x, c ; y ; \hbar)=\frac{1}{m ! n !} c^{i_{m}} \wedge \cdots \wedge c^{i_{1}} a_{i_{1} \ldots i_{m} j_{1} \ldots j_{n}}(x ; \hbar) \otimes y^{j_{n}} \vee \cdots \vee y^{j_{1}} .
$$

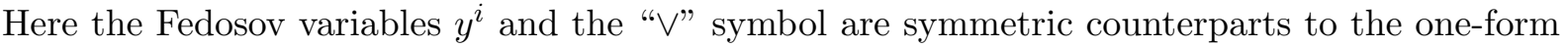
basis $c^{i} \equiv d x^{i}$ and the " $\wedge$ " symbol, i.e.,

$$
y^{j} \vee y^{i}=(-1)^{\varepsilon_{i} \varepsilon_{j}} y^{i} \vee y^{j} .
$$

We will be interested in covariant derivatives $\nabla_{i} a_{i_{1} \ldots i_{m} j_{1} \ldots j_{n}}$ of the above tensors. The covariant derivative $\nabla_{i}$ from equation (2.5) can be implemented on coordinate-free objects (4.2) by the following linear differential operator

$$
\nabla_{i}=\frac{\overrightarrow{\partial^{\ell}}}{\partial x^{i}}-\Gamma_{i}{ }_{j}{ }_{j} c^{j} \frac{\overrightarrow{\partial^{\ell}}}{\partial c^{k}}-\Gamma_{i}{ }^{k}{ }_{j} y^{j} \frac{\overrightarrow{\partial^{\ell}}}{\partial y^{k}} .
$$

If both the numbers of antisymmetric and symmetric indices are non-zero $m \neq 0 \wedge n \neq 0$, i.e., if the tensor $a_{i_{1} \ldots i_{m} j_{1} \ldots j_{n}}$ has mixed symmetry, the covariant derivative $\nabla_{i} a_{i_{1} \ldots i_{m} j_{1} \ldots j_{n}}$ will not belong to any of the $\mathcal{A}_{\text {.• }}$ spaces (4.1). We repair this by antisymmetrizing with respect to the indices $i, i_{1}, \ldots, i_{m}$. Such antisymmetrization can be implemented on coordinate-free objects (4.2) with the help of the following one-form valued Grassmann-even differential operator

$$
\nabla:=c^{i} \nabla_{i}=d-c^{i} \Gamma_{i}{ }^{k}{ }_{j} y^{j} \frac{\overrightarrow{\partial^{\ell}}}{\partial y^{k}},
$$

where we have followed common practice, and given the differential operator (4.4) the same name as the connection. In the second equality of equation (4.4) we have used that the connection is 
Table 1. Parities and gradings.

\begin{tabular}{|l|c||c|c|c|}
\hline$\downarrow$ Variable & Grading $\rightarrow$ & $\begin{array}{c}\text { Grassmann } \\
\text { parity }\end{array}$ & $\begin{array}{c}\text { Form } \\
\text { degree }\end{array}$ & $\begin{array}{c}\text { Fedosov } \\
\text { degree }\end{array}$ \\
\hline \hline Coordinates & $\downarrow$ Symbol $\rightarrow$ & $\varepsilon$ & $p$ & deg \\
\hline 1-form basis & $x^{i}$ & $\varepsilon_{i}$ & 0 & 0 \\
\hline Fedosov coordinates & $c^{i} \equiv d x^{i}$ & $\varepsilon_{i}$ & 1 & 0 \\
\hline Planck's constant & $y^{i}$ & $\varepsilon_{i}$ & 0 & 1 \\
\hline \hline Multiplicative structure & $\hbar$ & 0 & 0 & 2 \\
\hline Christoffel symbol & $m^{i j}$ & $\varepsilon_{i}+\varepsilon_{j}$ & 0 & even, $\geq 0$ \\
\hline Covariant derivative & $\Gamma^{k}{ }_{i j}$ & $\varepsilon_{i}+\varepsilon_{j}+\varepsilon_{k}$ & 0 & even, $\geq 0$ \\
\hline 1-form valued connection & $\nabla_{i}$ & $\varepsilon_{i}$ & 0 & even, $\geq 0$ \\
\hline de Rham exterior derivative & $\nabla \equiv c^{i} \nabla_{i}$ & 0 & 1 & even, $\geq 0$ \\
\hline Koszul-Tate differential & $d$ & 0 & 1 & 0 \\
\hline Contraction & $\delta=\{\varpi, \cdot\}$ & 0 & 1 & -1 \\
\hline Koszul-Tate Hamiltonian & $\delta^{*}=y^{j} i\left(\partial_{j}^{\ell}\right)$ & 0 & -1 & 1 \\
\hline Deformation 1-form & $\varpi \equiv c^{i} \omega_{i j} y^{j}$ & 0 & 1 & odd, $\geq 1$ \\
\hline Hamiltonian curvature 2-form & $r$ & 0 & 1 & $\geq 0$ \\
\hline & $\mathcal{R}$ & 0 & 2 & even, $\geq 2$ \\
\hline
\end{tabular}

torsion-free. (References [38, 37] consider a hybrid model where torsion is allowed in the $y$-sector but not in the $c$-sector in such a way that equation (4.4) remains valid.) Since the $\nabla$ operator is a first-order differential operator, i.e.,

$$
\nabla(a b)=(\nabla a) b+(-1)^{p_{a}} a(\nabla b),
$$

where $a$ and $b$ are two coordinate-free objects (4.2), it is customary to refer to $\nabla$ as a linear connection. (The order of the exterior factor $\bigwedge^{m}\left(T^{*} M\right)$ and the symmetric factor $\bigvee^{n}\left(T^{*} M\right)$ in expression (4.1) is opposite the standard convention to ease the use of covariant derivatives $\nabla$ that acts from the left.)

\section{The $\mathcal{A}$ algebra}

The direct sum

$$
\mathcal{A}:=\bigoplus_{m, n \geq 0} \mathcal{A}_{m n} \cong C^{\infty}(E)[[\hbar]]
$$

of the $\mathcal{A}_{m n}$ spaces (4.1) is an algebra with multiplication given by the tensor multiplication. It is both associative and commutative. As indicated in equation (5.1), the elements

$$
a=\oplus_{m, n \geq 0} a_{m n} \in \mathcal{A}, \quad a_{m n} \in \mathcal{A}_{m n},
$$

can be viewed as quantum functions $a=a(x, c ; y ; \hbar)$ on the Whitney sum

$$
E:=\Pi T M \oplus T M
$$

where $c^{i}$ are identified with the parity-inverted fiber coordinates for the fiber-wise parityinverted tangent bundle $\Pi T M$, and $y^{j}$ are identified with the fiber coordinates for the tangent bundle $T M$. The word "quantum function" just means that it is a formal power series in Planck's constant $\hbar$. 


\section{The Fedosov resolution degree}

The Fedosov degree "deg" is a (non-negative) integer grading of the $\mathcal{A}$ algebra defined as

$$
\operatorname{deg}\left(y^{i}\right)=1, \quad \operatorname{deg}(\hbar)=2,
$$

and zero for the two other remaining variables $x^{j}$ and $c^{k}$, cf. Table 1 . The Fedosov degree will play the rôle of resolution degree in the sense of homological perturbation theory $[29,7,27,1$, $2,25,35]$. We shall therefore often organize the algebra according to this grading:

$$
\mathcal{A}=\bigoplus_{n \geq 0} \mathcal{A}_{(n)}, \quad \mathcal{A}_{(n)}:=\{a \in \mathcal{A} \mid \operatorname{deg}(a)=n\} .
$$

Similarly, one may write the algebra element

$$
a=\oplus_{n \geq 0} a_{(n)} \in \mathcal{A}, \quad a_{(n)} \in \mathcal{A}_{(n)},
$$

as a direct sum of elements $a_{(n)}$ of definite Fedosov degree $\operatorname{deg}\left(a_{(n)}\right)=n$.

\section{The $\circ$ product}

One now builds an associative $\circ$ product $\mathcal{A} \times \mathcal{A} \rightarrow \mathcal{A}$ on the $\mathcal{A}$ algebra as a Moyal product $[34,41]$ in the $y$-variables,

$$
a \circ b:=\left(a \exp \left[\frac{\overleftarrow{\partial^{r}}}{\partial y^{j}} \frac{i \hbar}{2} m^{j k} \frac{\overrightarrow{\partial^{\ell}}}{\partial y^{k}}\right] b\right), \quad a, b \in \mathcal{A}
$$

The $\circ$ product is associative, because the $m^{j k}$-tensor is independent of $y$-variables. (The $y$ variables have been interpreted by Grigoriev and Lyakhovich [33, 5] as conversion variables for the conversion of second-class constraints into first-class $[3,4,6,28]$.$) The o product "preserves"$ the following gradings

$$
\begin{aligned}
& \operatorname{deg}(a \circ b) \geq \operatorname{deg}(a)+\operatorname{deg}(b), \\
& p(a \circ b)=p(a)+p(b), \\
& \varepsilon(a \circ b)=\varepsilon(a)+\varepsilon(b) .
\end{aligned}
$$

The connection $\nabla$ respects the o product,

$$
\nabla(a \circ b)=(\nabla a) \circ b+(-1)^{p_{a}} a \circ(\nabla b), \quad a, b \in \mathcal{A},
$$

as a consequence of the assumption (2.4).

\section{The Poisson bracket}

It is useful to define a Poisson bracket as

$$
\{a, b\}:=\left(a \frac{\overleftarrow{\partial^{r}}}{\partial y^{i}}\right) \omega^{i j}\left(\frac{\overrightarrow{\partial^{\ell}}}{\partial y^{j}} b\right), \quad a, b \in \mathcal{A} .
$$

(It should not be confused with the classical Poisson bracket (3.8).) The Poisson bracket (8.1) "preserves" the following gradings

$$
0 \leq \operatorname{deg}(\{a, b\}) \geq \operatorname{deg}(a)+\operatorname{deg}(b)-2,
$$




$$
\begin{aligned}
& p(\{a, b\})=p(a)+p(b), \\
& \varepsilon(\{a, b\})=\varepsilon(a)+\varepsilon(b) .
\end{aligned}
$$

The connection $\nabla$ respects the Poisson bracket:

$$
\nabla\{a, b\}=\{\nabla a, b\}+(-1)^{p_{a}}\{a, \nabla b\}, \quad a, b \in \mathcal{A} .
$$

\section{The o commutator}

The $\circ$ commutator is defined as

$$
[a \stackrel{\circ}{b}]:=a \circ b-(-1)^{\varepsilon_{a} \varepsilon_{b}+p_{a} p_{b}} b \circ a=i \hbar\{a, b\}+\mathcal{O}\left(\hbar^{2}\right), \quad a, b \in \mathcal{A} .
$$

Note the following useful observations:

- Each term in the o commutator $[a, b]$ contains at least one power of $\hbar$, so one may consider the fraction $\frac{1}{i \hbar}[a, b]$ without introducing negative powers of $\hbar$.

- The $\circ$ commutator $\frac{1}{i \hbar}[a, b]$ and the Poisson bracket $\{a, b\}$ are equal, if one of the entries $a$ or $b$ only contains terms with less than three $y$ 's.

The $\circ$ commutator may be expanded according to the Fedosov degree:

$$
[a, b]=\sum_{n \geq 0}[a \circ b]_{(n)},
$$

where

$$
\operatorname{deg}\left([a, b]_{(n)}\right)=\operatorname{deg}(a)+\operatorname{deg}(b)+n .
$$

\section{The Koszul-Tate differential $\delta$}

The Koszul-Tate differential is defined as

$$
\delta:=c^{i} \frac{\overrightarrow{\partial^{\ell}}}{\partial y^{i}}=\{\varpi, \cdot\}, \quad \operatorname{deg}(\delta)=-1 .
$$

In the second equality in equation (10.1) we have indicated that the Koszul-Tate differential is an inner derivation in the algebra $\left(\mathcal{A},+, \frac{1}{i \hbar}[\cdot, \cdot]\right)$ with generator

$$
\varpi:=c^{i} \omega_{i j} y^{j}=y^{j} c^{i} \tilde{\omega}_{i j} \in \mathcal{A}_{11}, \quad \operatorname{deg}(\varpi) \geq 1,
$$

which we shall refer to as the Hamiltonian for $\delta$. The Koszul-Tate differential $\delta$ will serve as the leading term in a resolution expansion of a deformed connection $D$, see Section 14 [29, 7, 27, 1, $2,25,35]$. Since the $\omega_{i j}$ tensor is covariantly preserved, cf. equation (3.4), it follows immediately that

$$
(\nabla \varpi)=-y^{j} c^{i} c^{k}\left(\nabla_{k} \tilde{\omega}_{i j}\right)=0
$$

even without using the skewsymmetry (3.1) or the antisymmetry (3.2) (or the torsion-free condition for that matter). As a corollary,

$$
[\nabla, \delta]=0 .
$$

The $\delta$-differential is nilpotent

$$
\delta^{2} \equiv \frac{1}{2}[\delta, \delta]=0
$$

and it respects the o product

$$
\delta(a \circ b)=(\delta a) \circ b+(-1)^{p_{a}} a \circ(\delta b) .
$$




\section{The Poincaré lemma and the homotopy operator $\delta^{-1}$}

There exists a version of the Poincaré lemma where the rôle of the de Rham exterior derivative $d \equiv c^{i} \partial^{\ell} / \partial x^{i}$ has been replaced by the Koszul-Tate differential $\delta \equiv c^{i} \partial^{\ell} / \partial y^{i}$, or equivalently, where the $x$-coordinates are replaced by the $y$-coordinates. As we shall see below, the equation

$$
(\delta b)=a, \quad a \in \mathcal{A},
$$

may be solved locally with respect to an algebra element $b \in \mathcal{A}$ whenever $a \in \mathcal{A}$ is a given $\delta$ closed algebra element with no 00-part $a_{00}=0$. (The 00-part $a_{00}$ is the part of the algebra element $a$ that is independent of the $c$ - and the $y$-variables, cf. Section 4.) In fact, a local solution $b$ to equation (11.1) may be extended to a global solution, since the total space is contractible in the $y$-directions. We shall use this crucial fact to guarantee the existence of global solutions to differential equations, whose differential operator is a deformation of the Koszul-Tate differential $\delta$, see Theorems 1 and 2. As usual when dealing with the Poincaré Lemma, it is useful to consider the inner contraction

$$
\delta^{*}:=y^{j} \frac{\overrightarrow{\partial^{\ell}}}{\partial c^{j}}=y^{j} i\left(\partial_{j}^{\ell}\right),
$$

which is dual to $\delta$ with respect to a $y \leftrightarrow c$ permutation. The commutator

$$
\left[\delta, \delta^{*}\right]=c^{i} \frac{\overrightarrow{\partial^{\ell}}}{\partial c^{i}}+y^{i} \frac{\overrightarrow{\partial^{\ell}}}{\partial y^{i}}
$$

is a Euler vector field that counts the number of $c$ 's and $y$ 's. The homotopy operator $\delta^{-1}$ is defined as

$$
\forall a \in \mathcal{A}_{m n}: \delta^{-1} a:=\left\{\begin{array}{lll}
\frac{1}{m+n}\left(\delta^{*} a\right) & \text { for } & (m, n) \neq(0,0), \\
0 & \text { for } \quad(m, n)=(0,0),
\end{array}\right.
$$

and extended by linearity to the whole algebra $\mathcal{A}$. The homotopy operator $\delta^{-1}$ is not a firstorder differential operator, in contrast to $\delta^{*}$. One easily obtains the following version of the Poincaré lemma.

Lemma 1 (Poincaré lemma). There is only non-trivial $\delta$-cohomology in the 00-sector with neither c's nor y's. A more refined statement is the following: For all $\delta$-closed elements $a \in \mathcal{A}$ with no 00-part, there exists a unique $\delta^{*}$-closed element $b \in \mathcal{A}$ with no 00-part, such that $a=(\delta b)$. Or equivalently, in symbols:

$$
\forall a \in \mathcal{A}:\left\{\begin{array}{c}
(\delta a)=0 \\
a_{00}=0
\end{array}\right\} \Rightarrow \exists ! b \in \mathcal{A}:\left\{\begin{array}{c}
a=(\delta b) \\
\left(\delta^{*} b\right)=0 \\
b_{00}=0
\end{array}\right\} .
$$

The unique element $b$ is given by the homotopy operator $\delta^{-1} a$.

\section{The Riemann curvature}

See [12] and [32] for related discussions. The Riemann curvature $R$ is defined as (half) the commutator of the $\nabla$ connection (2.6),

$$
R=\frac{1}{2}[\nabla \wedge \nabla]=-\frac{1}{2} d x^{j} \wedge d x^{i} \otimes\left[\nabla_{i}, \nabla_{j}\right]=-\frac{1}{2} d x^{j} \wedge d x^{i} \otimes \partial_{n}^{r} R_{i j k}^{n} \overrightarrow{d x^{k}},
$$




$$
R_{i j k}^{n}=\overrightarrow{d x^{n}}\left(\left[\nabla_{i}, \nabla_{j}\right] \partial_{k}^{r}\right)=(-1)^{\varepsilon_{n} \varepsilon_{i}}\left({\overrightarrow{\partial^{\ell}}}_{\partial x^{i}} \Gamma_{j k}^{n}\right)+\Gamma_{i m}^{n} \Gamma_{j k}^{m}-(-1)^{\varepsilon_{i} \varepsilon_{j}}(i \leftrightarrow j),
$$

where it is implicitly understood in the second equality of equation (12.1) that $\nabla_{i}$ does not act on $d x^{j}$. If $\nabla_{i}$ is supposed to act on $d x^{j}$, one should include a torsion-term

$$
d x^{i} \wedge\left[\nabla_{i}, d x^{k}\right] \nabla_{k}=-d x^{i} \wedge \Gamma_{i}{ }_{j}{ }_{j} d x^{j} \nabla_{k}=\frac{(-1)^{\varepsilon_{i}}}{2} T^{k}{ }_{i j} d x^{j} \wedge d x^{i} \nabla_{k}
$$

cf. equation (13.1). Note that the order of indices in the Riemann curvature tensor $R^{n}{ }_{i j k}$ is non-standard. This is to minimize appearances of Grassmann sign factors. Alternatively, the Riemann curvature tensor may be defined as

$$
R(X, Y) Z=\left(\left[\nabla_{X}, \nabla_{Y}\right]-\nabla_{[X, Y]}\right) Z=Y^{j} X^{i} R_{i j}{ }_{k} Z^{k} \partial_{n}^{\ell}
$$

where $X=X^{i} \partial_{i}^{\ell}, Y=Y^{j} \partial_{j}^{\ell}$ and $Z=Z^{k} \partial_{k}^{\ell}$ are vector fields of even Grassmann-parity. The Riemann curvature tensor $R_{i j}{ }^{n}{ }_{k}$ reads in local coordinates

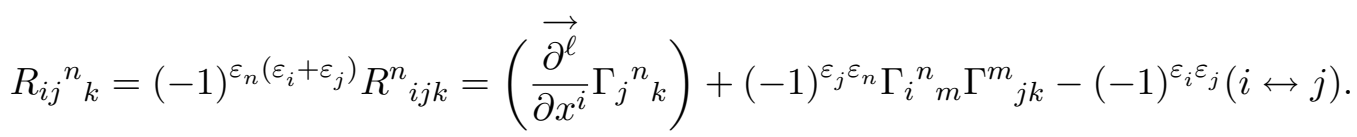

It is sometimes useful to reorder the indices in the Riemann curvature tensors as

$$
R_{i j k}^{n}=\left(\left[\nabla_{i}, \nabla_{j}\right] \partial_{k}^{\ell}\right)^{n}=(-1)^{\varepsilon_{k}\left(\varepsilon_{n}+1\right)} R_{i j}{ }^{n} .
$$

For a symplectic connection $\nabla$, we prefer to work with a $(0,4)$ Riemann tensor (as opposed to a $(1,3)$ tensor) by lowering the upper index with the symplectic metric (3.3). In terms of Christoffel symbols it is easiest to work with expression (12.2):

$$
\begin{aligned}
R_{n, i j k} & :=\omega_{n m} R^{m}{ }_{i j k}(-1)^{\varepsilon_{k}} \\
& =(-1)^{\varepsilon_{i} \varepsilon_{n}}\left(\frac{\overrightarrow{\partial^{\ell}}}{\partial x^{i}} \Gamma_{n, j k}+(-1)^{\varepsilon_{m}\left(\varepsilon_{i}+\varepsilon_{n}+1\right)+\varepsilon_{k}} \Gamma_{m, i n} \Gamma^{m}{ }_{j k}\right)-(-1)^{\varepsilon_{i} \varepsilon_{j}}(i \leftrightarrow j) .
\end{aligned}
$$

In the second equality of equation (12.5) we have use the symplectic condition (3.4). If we use the symplectic condition (3.4) one more time on the first term in equation (12.5), we derive the following symmetry

$$
R_{n, i j k}=(-1)^{\left(\varepsilon_{i}+\varepsilon_{j}\right)\left(\varepsilon_{k}+\varepsilon_{n}\right)+\varepsilon_{k} \varepsilon_{n}}(k \leftrightarrow n) .
$$

This symmetry becomes clearer if we instead start from expression (12.4) and define

$$
R_{i j, k n}:=R_{i j k}{ }^{m} \tilde{\omega}_{m n}=-(-1)^{\varepsilon_{n}\left(\varepsilon_{i}+\varepsilon_{j}+\varepsilon_{k}\right)} R_{n, i j k} .
$$

Then the symmetry (12.6) simply translates into a symmetry between the third and fourth index:

$$
R_{i j, k n}=(-1)^{\varepsilon_{k} \varepsilon_{n}}(k \leftrightarrow n) .
$$

We note that the torsion-free condition has not been used at all in this Section 12. 


\section{The curvature two-form $R$}

Let us now calculate the commutator of two $\nabla_{i}$ operators using the realization (4.3) of the covariant derivative:

$$
-\left[\nabla_{i}, \nabla_{j}\right]=R_{i j}{ }_{k} c^{k} \frac{\overrightarrow{\partial^{\ell}}}{\partial c^{n}}+R_{i j}{ }^{n}{ }_{k} y^{k} \frac{\overrightarrow{\partial^{\ell}}}{\partial y^{n}}=R_{i j}{ }_{k}{ }^{k}{ }^{k} \frac{\overrightarrow{\partial^{\ell}}}{\partial c^{n}}+\frac{1}{2}(-1)^{\varepsilon_{k}+\varepsilon_{n}}\left\{R_{i j, k n} y^{n} y^{k}, \cdot\right\} .
$$

Using realization (4.3) the curvature two-form reads

$$
\begin{aligned}
R & :=\nabla^{2}=c^{i}\left[\nabla_{i}, c^{k}\right] \nabla_{k}-\frac{1}{2} c^{j} c^{i}\left[\nabla_{i}, \nabla_{j}\right] \\
& =\frac{(-1)^{\varepsilon_{i}}}{2} T^{k}{ }_{i j} c^{j} c^{i} \nabla_{k}+\frac{1}{2} c^{k} c^{j} c^{i} R_{i j k}{ }^{n} \frac{\overrightarrow{\partial^{\ell}}}{\partial c^{n}}+\frac{1}{2} y^{k} c^{j} c^{i} R_{i j k}{ }^{n} \frac{\overrightarrow{\partial^{\ell}}}{\partial y^{n}} \\
& =\frac{1}{2} y^{k} c^{j} c^{i} R_{i j, k n}(-1)^{\varepsilon_{n}}\left\{y^{n}, \cdot\right\}=\{\mathcal{R}, \cdot\},
\end{aligned}
$$

where

$$
\mathcal{R}:=\frac{1}{4} y^{n} y^{k} c^{j} c^{i} R_{i j, k n} \in \mathcal{A}_{22}
$$

is a Hamiltonian for the curvature two-form $R$. In this formalism the connection $\nabla_{i}$ is supposed to act on $c^{k} \equiv d x^{k}$, so the torsion-term (12.3) is included in the curvature two-form (13.1). In the fourth equality of equation (13.1) we use the torsion-free condition (3.6) and the first Bianchi identity for a torsion-free connection

$$
0=\sum_{i, j, k \text { cycl. }}(-1)^{\varepsilon_{i} \varepsilon_{k}} R_{i j k}^{n}
$$

to ensure that the $x$ - and $c$-derivative term in equation (13.1) vanishes. As we shall see below, it is vital that there is no $x$ - and $c$-derivative term in the $R$ curvature (13.1). This is the main reason why the connection $\nabla$ is assumed to be torsion-free. (See also the comment after equation (16.1).) The first Bianchi identity (13.2) also implies that the Hamiltonian curvature two-form $\mathcal{R}$ is $\delta$-closed:

$$
(\delta \mathcal{R})=\frac{1}{2} c^{k} c^{j} c^{i} R_{i j, k n} y^{n}(-1)^{\varepsilon_{n}}=0 .
$$

Similarly, the second Bianchi identity for a torsion-free connection

$$
0=\sum_{i, j, k \text { cycl. }}(-1)^{\varepsilon_{j} \varepsilon_{k}} \nabla_{k} R_{i j}{ }^{n} m
$$

implies that the $\nabla$ operator preserves the Hamiltonian curvature two-form $\mathcal{R}$ :

$$
(\nabla \mathcal{R})=\frac{1}{4} c^{j} c^{i} c^{k}\left(\nabla_{k} R_{i j, n m}\right) y^{m} y^{n}(-1)^{\varepsilon_{n}+\varepsilon_{m}}=0
$$

\section{Higher-order $D$ connection}

We next deform the linear $\nabla$ connection from equation (4.4) into a higher-order connection $D: \Gamma(T M)[[\hbar]] \times \mathcal{A} \rightarrow \mathcal{A}$,

$$
D:=\nabla-\delta+\frac{1}{i \hbar}[r \stackrel{\circ}{,}]=\nabla+\frac{1}{i \hbar}[r-\varpi \stackrel{\circ}{\cdot}]=\sum_{n \geq-1} \stackrel{(n)}{D}, \quad \operatorname{deg}(\stackrel{(n)}{D})=n,
$$


with the help of a so-called deformation one-form

$$
r=\oplus_{n \geq 0} r_{(n)} \in \mathcal{A}_{1 \bullet}, \quad \operatorname{deg}\left(r_{(n)}\right)=n .
$$

As we soon shall see, it is better to think of $D$ as a deformation of (minus) the Koszul-Tate differential $\delta$ rather than the connection $\nabla$. The word "higher-order" refers to that $D$ is not necessarily a linear derivation of the tensor algebra $(\mathcal{A},+, \otimes)$, cf. equation (4.5). However, it is a linear derivation of the $\circ$ algebra $(\mathcal{A},+, \circ)$, similar to equations (7.2) and (10.3). The $\nabla$ and $D$ connections may be expanded in Fedosov degree:

$$
\nabla=\sum_{n \geq 0} \stackrel{(n)}{\nabla}, \quad \operatorname{deg}(\stackrel{(n)}{\nabla})=n, \quad \stackrel{\text { (odd) }}{\nabla}=0,
$$

and

$$
\stackrel{(n)}{D}:= \begin{cases}\frac{1}{i \hbar}\left[r_{(1)}, \cdot \cdot\right]_{(0)}-\delta & \text { for } n=-1, \\ \stackrel{(n)}{\nabla}+\frac{1}{i \hbar} \sum_{k=0}^{n+2}\left[r_{(k)} \stackrel{\circ}{,} \cdot\right]_{(n+2-k)} & \text { for } n \geq 0 .\end{cases}
$$

Note that the connection $D$ does not depend on $r_{(0)}$. Also note that it will be necessary to assume that the (1)-sector vanishes

$$
r_{(1)}=0
$$

to ensure that (minus) the Koszul-Tate differential $\delta$ is the sole leading term in the $D$ expansion (14.1).

\section{The $R_{D}$ curvature}

The curvature two-form $R_{D}$ for the $D$ connection is

$$
\begin{aligned}
& R_{D}:=D^{2}=\left(\nabla+\frac{1}{i \hbar}[r-\varpi, \cdot]\right)^{2} \\
& =\nabla^{2}+\frac{1}{i \hbar}[\nabla(r-\varpi), \cdot]+\frac{1}{(i \hbar)^{2}}[r-\varpi,[r-\varpi, \cdot]]=\frac{1}{i \hbar}\left[\mathcal{R}_{D}, \cdot\right],
\end{aligned}
$$

where the Hamiltonian $\mathcal{R}_{D}$ is

$$
\mathcal{R}_{D}:=\mathcal{R}+\nabla(r-\varpi)+\frac{1}{2 i \hbar}[r-\varpi \circ r-\varpi]=\mathcal{R}+(\nabla-\delta) r+\frac{1}{2 i \hbar}[r \circ r]-\omega .
$$

In the third and fourth equality of equation (15.1) we use that $\nabla$ respects the $\circ$ product (7.2) and the Jacobi identity for the $\circ$ product, respectively. In the second equality of equation (15.2) we use that $(\nabla \varpi)=0, \delta=\{\varpi, \cdot\}$ and $\{\varpi, \varpi\}=-2 \omega$.

\section{Flat/nilpotent $D$ connection}

The next main principle of Fedosov quantization is to choose the $D$ connection to be flat, or equivalently, nilpotent:

$$
R_{D} \equiv D^{2} \equiv \frac{1}{2}[D, D]=0 .
$$


In other words, the odd $D$ operator is a deformation of the odd Koszul-Tate differential $\delta$, such that the nilpotency is preserved, cf. equations (10.2) and (16.1). (This setup is similar to the construction of an odd nilpotent BRST operator [29, 7, 27, 1, 2, 25, 35].) Since we want to achieve the nilpotency (16.1), it now becomes clear why it was so important that the $x$ and $c$-derivative terms in the $R$ curvature two-form (13.1) vanish. This is because there are no other $x$ - and $c$-derivatives in the $R_{D}$ curvature two-form (15.1) to cancel them. (All other derivatives in equation (15.1) are $y$-derivatives.) This crucial point is the main reason that the $\nabla$ connection is assumed to be torsion-free. (References $[38,37]$ consider a hybrid model where torsion is allowed in the $y$-sector but not in the $c$-sector to avoid the $x$ - and $c$-derivatives. Note, however, that they restrict the possible torsion by imposing both the independent conditions $\left(\nabla_{i} \tilde{\omega}_{j k}\right)=0$ and $(d \omega)=0$ at the same time.) For the curvature two-form $R_{D}$ to be zero, it is enough to let the Hamiltonian curvature two-form $\mathcal{R}_{D}$ be Abelian, i.e., to let it belong to the center

$$
Z(\mathcal{A}):=\{a \in \mathcal{A} \mid[\mathcal{A} ; a]=0\}=\{a \in \mathcal{A} \mid\{\mathcal{A}, a\}=0\}=\mathcal{A}_{\bullet 0} \equiv \Gamma\left(\bigwedge^{\bullet}\left(T^{*} M\right)\right)[[\hbar]]
$$

of the algebra $(\mathcal{A},+, \circ)$. In other words, there should exist an Abelian two-form $C \in \mathcal{A}_{20}$, such that

$$
\mathcal{R}_{D}=-C-\omega \in \mathcal{A}_{20}
$$

Here $\omega$ is just the symplectic two-form (3.3) itself. (Recall that $\omega$ trivially belongs to the center $Z(\mathcal{A})$. The signs and the shift with $\omega$ in equation (16.2) are introduced without loss of generality for later convenience.) The Abelian condition (16.2) turns into Fedosov's $r$-equation

$$
(\delta r)=\mathcal{R}+C+(\nabla r)+\frac{1}{2 i \hbar}[r \stackrel{\circ}{,} r] .
$$

Theorem 1 (Fedosov's 1st theorem). Let there be given an Abelian two-form $C \in \mathcal{A}_{20}$ that is closed (三symplectic),

$$
(d C)=0
$$

and that satisfies the boundary condition

$$
C_{(0)}=0
$$

Then there exists a unique one-form valued $r$-solution

$$
r=\oplus_{n \geq 0} r_{(n)} \in \mathcal{A}_{1 \bullet}, \quad \operatorname{deg}\left(r_{(n)}\right)=n,
$$

to Fedosov's equation (16.3) such that $r$ is $\delta^{*}$-closed,

$$
\left(\delta^{*} r\right)=0
$$

and satisfies the boundary condition

$$
r_{(1)}=0
$$

for the (1)-sector. As a consequence, it turns out that the first three sectors $r_{(0)}, r_{(1)}$ and $r_{(2)}$ are identically zero. 
Proof. Let us split the Abelian condition (16.3) in Fedosov degree:

$$
\begin{aligned}
\delta r_{(0)} \equiv & 0, \\
\delta r_{(n+1)}= & \mathcal{R}_{(n)}+C_{(n)}+\sum_{k=0}^{n} \stackrel{(n-k)}{\nabla} r_{(k)} \\
& +\frac{1}{2 i \hbar} \sum_{0 \leq k, \ell}^{k+\ell \leq n+2}\left[r_{(k)},{ }^{\circ} r_{(\ell)}\right]_{(n+2-k-\ell)} \quad \text { for } n \geq 0 .
\end{aligned}
$$

In equation (16.8) the Hamiltonian curvature two-form $\mathcal{R}$ and the closed Abelian two-form $C$ have also been expanded in Fedosov degree

$$
\begin{array}{lll}
\mathcal{R}=\oplus_{n \geq 2} \mathcal{R}_{(n)} \in \mathcal{A}_{22}, & \operatorname{deg}\left(\mathcal{R}_{(n)}\right)=n, & \mathcal{R}_{(\text {odd })}=0, \\
C=\oplus_{n \geq 0} C_{(n)} \in \mathcal{A}_{20}, & \operatorname{deg}\left(C_{(n)}\right)=n, & C_{(\text {odd })}=0 .
\end{array}
$$

A priori it is known that the (0)-sector $r_{(0)}=c^{i} \eta_{i}(x)$ is a $y$ - and $\hbar$-independent one-form. The equation (16.7) is therefore automatically satisfied. It follows from equation (16.5) that

$$
0=\delta^{*} r_{(0)}=y^{i} \eta_{i}(x)
$$

Therefore the (0)-sector

$$
r_{(0)}=0
$$

vanishes identically. Equation (16.8) with $n=0$ is automatically satisfied because of the two boundary conditions (16.4) and (16.6). Putting $n=1$ in equation (16.8) yields $\delta r_{(2)}=0$. Hence the $(2)$-sector $r_{(2)}$ is a one-form that is both $\delta$-closed and $\delta^{*}$-closed, and therefore it must be identically zero as well:

$$
r_{(2)}=0 \text {. }
$$

Since $r_{(0)}, r_{(1)}$ and $r_{(2)}$ are zero, the right-hand side expression for $\delta r_{(n+1)}$ in equation (16.8) will only depend on previous entries $r_{(\leq n)}$. Hence equation (16.8) is a recursive relation. The consistency relation for the Abelian condition (16.3) is that the right-hand side should be $\delta$ closed. This is indeed the case:

$$
\begin{aligned}
\delta(\mathrm{RHS}) & =\delta\left(\mathcal{R}+C+\nabla r+\frac{1}{2 i \hbar}[r \circ r]\right)=\delta \mathcal{R}+[\delta, \nabla] r-\nabla(\delta r)+\frac{1}{i \hbar}[\delta r \circ r]=-D(\delta r) \\
& =-\nabla\left(\mathcal{R}+C+\nabla r+\frac{1}{2 i \hbar}[r \circ r]\right)+\frac{1}{i \hbar}\left[\mathcal{R}+C+\nabla r+\frac{1}{2 i \hbar}[r \circ r] \circ, r\right]=0 .
\end{aligned}
$$

In the second equality of equation (16.9) we have used that $\delta$ respects the o product. In the third equality we have used that $(\delta \mathcal{R})=0$, that $[\delta, \nabla]=0$, and that $\delta$ is nilpotent. In the fifth (=last) equality we have used that $(\nabla \mathcal{R})=0$, that $(d C)=0$, that $\nabla^{2}=\{\mathcal{R}, \cdot\}$, that $\nabla$ respects the $\circ$ product, and the Jacobi identity for the o product.

We now prove by induction on the Fedosov degree $(n)$ that there exists a unique solution $r_{(n+1)}$ to equation (16.8) if there exists a unique solution for all the previous entries $r_{(\leq n)}$. This is essentially a consequence of the Poincaré lemma 1. Uniqueness: The difference

$$
\Delta r_{(n+1)}:=r_{(n+1)}^{\prime}-r_{(n+1)}^{\prime \prime}
$$


between two solutions $r_{(n+1)}^{\prime}$ and $r_{(n+1)}^{\prime \prime}$ must satisfy the homogeneous version $\delta\left(\Delta r_{(n+1)}\right)=0$ of equation (16.8), i.e., with no right-hand side. Hence the difference $\Delta r_{(n+1)}$ is a one-form that is both $\delta$-closed and $\delta^{*}$-closed, and therefore it must be identically zero. Existence: Define

$$
r_{(n+1)}:=\delta^{-1}\left(\operatorname{RHS}_{(n)}\right) \quad \text { for } \quad n \geq 0,
$$

where $\mathrm{RHS}_{(n)}$ is the two-form valued right-hand side of equation (16.8). This clearly defines a $\delta^{*}$-closed one-form $r_{(n+1)}$. To check equation (16.8), it is enough to check that the two-form $\operatorname{RHS}_{(n)}$ is $\delta$-closed. But this follows by linearity from the consistency relation (16.9), because $\nabla$ and $\circ$ both carry positive Fedosov degree, and the first three $r$-sectors vanish to cancel the negative Fedosov degree coming from the $\hbar^{-1}$-factor, so that only previous entries $r_{(\leq n)}$ can participate to the $(n)$-sector.

We emphasize that the unique deformation one-form $r$ from Fedosov's 1st theorem is globally well-defined, since it basically appeared from inverting the Koszul-Tate $\delta$ differential, cf. Section 11. Normally, one would choose a trivial Abelian two-form $C \equiv 0$. Also note that the two-form $\mathcal{R}+C$ is the lone source term that forces $r$ to be non-trivial. We list here the first few unique $r$-terms:

$$
\begin{aligned}
& r_{(0)}=0, \quad r_{(1)}=0, \quad r_{(2)}=0, \quad r_{(3)}=\delta^{-1}\left(\mathcal{R}_{(2)}+C_{(2)}\right), \\
& r_{(4)}=\delta^{-1}\left(\stackrel{(0)}{\nabla} r_{(3)}\right), \quad \ldots
\end{aligned}
$$

Similarly, the first few terms in the $D$ expansion read

$$
\stackrel{(-1)}{D}=-\delta, \quad \stackrel{(0)}{D}=\stackrel{(0)}{\nabla}, \quad \stackrel{(1)}{D}=\frac{1}{i \hbar}\left[r_{(3)} \stackrel{\circ}{\cdot} \cdot\right]_{(0)}, \quad \ldots
$$

\section{Horizontal sections}

Fedosov's 1st theorem establishes the existence of a globally well-defined, unique, flat/nilpotent $D$ connection. Since this higher-order connection $D$ is flat, it is possible to solve the horizontal condition $(D a)=0$ locally for a zero-form valued section $a \in \mathcal{A}_{0} \bullet$. (In other words, the flatness relation (16.1) is the local consistency relation for the horizontal condition.) As we shall see below there is no obstruction in patching together local horizontal sections $a$ into global horizontal sections, basically because $D$ is a deformation of (minus) the Koszul-Tate $\delta$-differential.

Theorem 2 (Fedosov's 2nd theorem). Let there be given a quantum function (also known as a symbol) $f \in C^{\infty}(M)[[\hbar]] \equiv \mathcal{A}_{00}$. Then there exists a unique zero-form valued section

$$
a=\oplus_{n \geq 0} a_{(n)} \in \mathcal{A}_{0 \bullet}, \quad \operatorname{deg}\left(a_{(n)}\right)=n,
$$

that is D-horizontal

$$
(D a)=0
$$

and that satisfies the boundary condition

$$
\left.a\right|_{y=0} \equiv a_{00}=f .
$$

Proof. First note that that a zero-form $a \in \mathcal{A}_{0}$ • is automatically $\delta^{*}$-closed:

$$
\left(\delta^{*} a\right) \equiv 0, \quad a \in \mathcal{A}_{0 \bullet} .
$$


The horizontal condition $(D a)=0$ becomes

$$
(\delta a)=(\nabla a)+\frac{1}{i \hbar}[r, a] .
$$

Let us split the horizontal condition (17.2) in Fedosov degree:

$$
\begin{aligned}
& \delta a_{(0)} \equiv 0, \\
& \delta a_{(n+1)}=\sum_{k=0}^{n} \stackrel{(n-k)}{\nabla} a_{(k)}+\frac{1}{i \hbar} \sum_{0 \leq k, \ell}^{k+\ell \leq n+2}\left[r_{(k)}, a_{(\ell)}\right]_{(n+2-k-\ell)} \quad \text { for } n \geq 0 .
\end{aligned}
$$

Note that the right-hand side expression for $\delta a_{(n+1)}$ only depends on previous entries $a_{(\leq n)}$, because $r_{(0)}, r_{(1)}$ and $r_{(2)}$ are zero. Hence equation (17.3) is a recursive relation. The consistency relation for the horizontal condition (17.2) is that the right-hand side should be $\delta$-closed. This is indeed the case:

$$
\begin{aligned}
& \delta(\mathrm{RHS})=\delta\left(\nabla a+\frac{1}{i \hbar}[r \stackrel{\circ}{;} a]\right)=[\delta, \nabla] a-\nabla(\delta a)-\frac{1}{i \hbar}[r \stackrel{\circ}{\delta} \delta a]+\frac{1}{i \hbar}[\delta r ; a] \\
& =-\nabla\left(\nabla a+\frac{1}{i \hbar}[r \stackrel{\circ}{a} a]\right)-\frac{1}{i \hbar}\left[r \stackrel{\circ}{i \hbar} a+\frac{1}{i \hbar}[r, a]\right]
\end{aligned}
$$

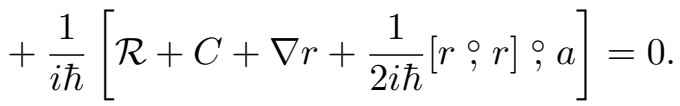

In the second equality of equation (17.4) we have used that $\delta$ respects the o product. In the third equality we have used that $[\delta, \nabla]=0$. In the fourth (=last) equality we have used that $\nabla^{2}=\{\mathcal{R}, \cdot\}$, that $\nabla$ respects the $\circ$ product, and the Jacobi identity for the o product.

We now prove by induction on the Fedosov degree $(n)$ that there exists a unique solution $a_{(n+1)}$ to equation (17.3) if there exists a unique solution for all the previous entries $a_{(\leq n)}$. This is essentially a consequence of the Poincaré lemma 1. Uniqueness: The difference

$$
\Delta a_{(n+1)}:=a_{(n+1)}^{\prime}-a_{(n+1)}^{\prime \prime}
$$

between two solutions $a_{(n+1)}^{\prime}$ and $a_{(n+1)}^{\prime \prime}$ must satisfy the homogeneous versions $\delta\left(\Delta a_{(n+1)}\right)=0$ and $\Delta a_{(n+1) 00}=0$ of the horizontal condition (17.3) and boundary condition (17.1), i.e., with no right-hand sides. Hence the difference $\Delta a_{(n+1)}$ is both $\delta$-closed, $\delta^{*}$-closed and with no 00-sector. Therefore it must be identically zero. Existence: Define

$$
\begin{aligned}
& a_{(0)}:=f_{(0)}, \\
& a_{(n+1)}:=f_{(n+1)}+\delta^{-1}\left(\operatorname{RHS}_{(n)}\right) \quad \text { for } \quad n \geq 0,
\end{aligned}
$$

where $\mathrm{RHS}_{(n)}$ is the one-form valued right-hand side of equation (17.3). This clearly defines a zero-form $a_{(n+1)}$ that satisfies the boundary condition (17.1). To check equation (17.3), it is enough to check that the one-form $\mathrm{RHS}_{(n)}$ is $\delta$-closed. But this follows by linearity from the consistency relation (17.4), because $\nabla$ and $\circ$ both carry positive Fedosov degree, and the first three $r$-sectors vanish to cancel the negative Fedosov degree coming from the $\hbar^{-1}$-factor, so that only previous entries $a_{(\leq n)}$ can participate to the $(n)$-sector.

We list here the solution to the unique first-order correction $a_{(1)}$ :

$$
a_{(1)}-f_{(1)}=\delta^{-1}\left(\stackrel{(0)}{\nabla} a_{(0)}\right)=y^{i}\left(\frac{\overrightarrow{\partial^{\ell}}}{\partial x^{i}} f_{(0)}\right),
$$

which we'll use in the next Section 18. 


\section{The $*$ product}

Fedosov's 2nd theorem establishes an isomorphism

$$
\left(\mathcal{A}_{00},+, *\right) \ni f \stackrel{Q}{\longmapsto} Q(f) \in\left(W_{D},+, \circ\right)
$$

between the algebra $\mathcal{A}_{00}$ of quantum functions (=symbols),

$$
\mathcal{A}_{00}=C^{\infty}(M)[[\hbar]]=\left\{a \in \mathcal{A} \mid(\delta a)=0=\left(\delta^{*} a\right)\right\},
$$

and the algebra of zero-form valued horizontal sections,

$$
W_{D}:=\left\{a \in \mathcal{A}_{0 \bullet} \mid(D a)=0\right\} .
$$

That the vector space $\left(W_{D},+, \circ\right)$ is an subalgebra, i.e., closed with respect to the o product, follows basically because the connection $\nabla$ respects the $\circ$ product, cf. equation (7.2). The $*$ product in diagram (18.1) is by definition induced from the $\circ$ product as

$$
f * g:=Q^{-1}(Q(f) \circ Q(g)) .
$$

This * product obviously inherits associativity from the o product, and the $Q$ map (18.1) is obviously an algebra isomorphism. Moreover, the inverse map

$$
\left.\left(W_{D},+, \circ\right) \ni a \stackrel{Q^{-1}}{\mapsto} a\right|_{y=0} \equiv a_{00} \in\left(\mathcal{A}_{00},+, *\right)
$$

is simply the restriction to $y=0$, cf. boundary condition (17.1). It remains to check that the classical boundary condition (2.1) holds. Let us first expand to the appropriate orders:

$$
\begin{aligned}
& Q(f)=a_{(0)}+a_{(1)}+a_{(2)}+\mathcal{O}\left(\hbar^{2}, y \hbar, y^{3}\right), \\
& Q(g)=b_{(0)}+b_{(1)}+b_{(2)}+\mathcal{O}\left(\hbar^{2}, y \hbar, y^{3}\right), \\
& Q(f) \circ Q(g)=Q(f) Q(g)+\frac{i \hbar}{2} Q(f) \frac{\partial^{r}}{\partial y^{j}} m^{j k} \frac{\overrightarrow{\partial^{\ell}}}{\partial y^{k}} Q(g)+\mathcal{O}\left(\hbar^{2}\right) .
\end{aligned}
$$

Therefore

$$
\begin{aligned}
& \lim _{\hbar \rightarrow 0} f * g=f_{(0)} g_{(0)}, \\
& \lim _{\hbar \rightarrow 0} \frac{f * g-f_{(0)} g_{(0)}}{i \hbar}=\frac{\left.a_{(2)}\right|_{y=0}}{i \hbar} b_{(0)}+a_{(0)} \frac{\left.b_{(2)}\right|_{y=0}}{i \hbar}+\frac{1}{2} a_{(1)} \frac{\overleftarrow{\partial^{r}}}{\partial y^{j}} m_{(0)}^{j k} \frac{\overrightarrow{\partial^{\ell}}}{\partial y^{k}} b_{(1)},
\end{aligned}
$$

or equivalently,

$$
\lim _{\hbar \rightarrow 0} \frac{f * g-f g}{i \hbar}=\frac{1}{2} f_{(0)} \frac{\overleftarrow{\partial^{r}}}{\partial x^{j}} m_{(0)}^{j k} \frac{\overrightarrow{\partial^{\ell}}}{\partial x^{k}} g_{(0)}
$$

Equations (18.2) and (18.3) are precisely the content of the classical boundary condition (2.1). It is also easy to check condition (2.2).

Note added. The author has kindly been informed by a referee of the existence of the Ph.D. Thesis [20], which the author has been unable to obtain, and which considers Fedosov quantization on supermanifolds. 


\section{Acknowledgement}

The author thanks I.A. Batalin, D. Sternheimer and the three referees for comments. The work of K.B. is supported by the Ministry of Education of the Czech Republic under the project MSM 0021622409.

Editorial Comments. This paper presents the Fedosov construction in a fairly general framework. It is generally known that the tensor need not be skew-symmetric as long as its skewsymmetric part is non-degenerate, can be a series in the deformation parameter, and that Fedosov's construction has also been extended to the case of super-manifolds. However it appears from the last reports that there is a point in publishing the unified presentation of the author, which should be viewed as a good review of Fedosov's construction in a quite general context, even if the author overemphasizes somewhat the importance of the generalizations he considers.

\section{References}

[1] Batalin I.A., Fradkin E.S., A generalized canonical formalism and quantization of reducible gauge theories, Phys. Lett. B 122 (1983), 157-164.

[2] Batalin I.A., Fradkin E.S., Operator quantization of relativistic dynamical systems subject to first class constraints, Phys. Lett. B 128 (1983), 303-308.

Batalin I.A., Fradkin E.S., Operator quantization and abelization of dynamical systems subject to first-class constraints, Riv. Nuovo Cimento 9 (1986), 1-48.

[3] Batalin I.A., Fradkin E.S., Operatorial quantization of dynamical systems subject to second class constraints, Nuclear Phys. B 279 (1987), 514-528.

Batalin I.A., Fradkin E.S., Operator quantization of dynamical systems with irreducible first and second class constraints, Phys. Lett. B 180 (1986), 157-162, Erratum, Phys. Lett. B 236 (1990), 528.

[4] Batalin I.A., Fradkin E.S., Fradkina T.E., Another version for operatorial quantization of dynamical systems with irreducible constraints, Nuclear Phys. B 314 (1989), 158-174, Erratum, Nuclear Phys. B 323 (1989), 734-735.

Batalin I.A., Fradkin E.S., Fradkina T.E., Generalized canonical quantization of dynamical systems with constraints and curved phase space, Nuclear Phys. B 332 (1990), 723-736.

[5] Batalin I.A., Grigoriev M.A., Lyakhovich S.L., Star product for second class constraint systems from a BRST theory, Theoret. and Math. Phys. 128 (2001), 1109-1139, hep-th/0101089.

[6] Batalin I.A., Tyutin I.V., An Sp(2) covariant formalism of a generalized canonical quantization with second class constraints, Internat. J. Modern Phys. A 6 (1991), 3599-3612.

[7] Batalin I.A., Vilkovisky G.A., Relativistic $S$ matrix of dynamical systems with boson and fermion constraints, Phys. Lett. B 69 (1977), 309-312.

[8] Bayen F., Flato M., Fronsdal C., Lichnerowicz A., Sternheimer D., Deformation theory and quantization. I. Deformations of symplectic structures, Ann. Physics 111 (1978), 61-110.

Bayen F., Flato M., Fronsdal C., Lichnerowicz A., Sternheimer D., Deformation theory and quantization. II. Physical applications, Ann. Physics 111 (1978), 111-151.

[9] Berezin F.A., Quantization, Math. USSR-Izv. 38 (1974), 1109-1165.

[10] Berezin F.A., General concept of quantization, Comm. Math. Phys. 40 (1975), 153-174.

[11] Berezin F.A., Quantization in complex symmetric spaces, Math. USSR-Izv. 39 (1975), 341-379.

[12] Bering K., Almost parity structure, connections and vielbeins in BV geometry, physics/9711010.

[13] Bordemann M., The deformation quantization of certain super-Poisson brackets and BRST cohomology, in Conférence Moshé Flato 1999, Quantization, Deformation, and Symmetries, Vol. II, Editors G. Dito and D. Sternheimer, Math. Phys. Stud., Vol. 22, Kluwer Acad. Publ., Dordrecht, 2000, 45-68, math.QA/0003218.

[14] Bordemann M., Waldmann S., A Fedosov star product of the Wick type for Kähler manifolds, Lett. Math. Phys. 41 (1997), 243-253, q-alg/9605012.

[15] Cattaneo A., Felder G., Tomassini L., From local to global deformation quantization of Poisson manifolds, Duke Math. J. 115 (2002) 329-352, math.QA/0012228. 
[16] Cattaneo A., Felder G., Tomassini L., Fedosov connections on jet bundles and deformation quantization, in Deformation Quantization (Strasbourg, 2001), IRMA Lect. Math. Theor. Phys., Vol. 1, de Gruyter, Berlin, 2002 191-202, math.QA/0111290.

[17] Dirac P.A.M., The fundamental equations of quantum mechanics, Proc. Roy. Soc. London A 109 (1925), 642-653.

[18] Dolgushev V.A., Lyakhovich S.L., Sharapov A.A., Wick type deformation quantization of Fedosov manifolds, Nuclear Phys. B 606 (2001), 647-672, hep-th/0101032.

[19] Donin J., Classification of polarized deformation quantizations, J. Geom. Phys. 48 (2003), 546-579, math.QA/0205211.

[20] Eckel R.K., Quantisierung von Supermannigfaltigkeiten à la Fedosov, Ph.D. Thesis, University of Freiburg, 2000.

[21] Fedosov B.V., Formal quantization, in Some Problems in Modern Mathematics and Their Applications to Problems in Mathematical Physics, Editor L.D. Kudryavtsev, Moscow Phys.-Tech. Inst., 1985, 129-136.

[22] Fedosov B.V., Quantization and the index, Sov. Phys. Dokl. 31 (1986), 877-878.

[23] Fedosov B.V., A simple geometrical construction of deformation quantization, J. Differential Geom. 40 (1994), 213-238.

[24] Fedosov B.V., Deformation quantization and index theory, Mathematical Topics, Vol. 9, Akademie Verlag, Berlin, 1996.

[25] Fisch J., Henneaux M., Stasheff J., Teitelboim C., Existence, uniqueness and cohomology of the classical BRST charge with ghosts of ghosts, Comm. Math. Phys. 120 (1989), 379-407.

[26] Farkas D.R., A ring-theorist's description of Fedosov quantization, Lett. Math. Phys. 51 (2000), 161-177, math.SG/0004071.

[27] Fradkin E.S., Fradkina T.E., Quantization of relativistic systems with boson and fermion first and second class constraint, Phys. Lett. B 72 (1978), 343-348.

[28] Fradkin E.S., Linetsky V.Ya., BFV approach to geometric quantization, Nuclear Phys. B 431 (1994) 569621.

Fradkin E.S., Linetsky V.Ya., BFV quantization on Hermitian symmetric spaces, Nuclear Phys. B 444 (1995), 577-601.

[29] Fradkin E.S., Vilkovisky G.A., Quantization of relativistic systems with constraints, Phys. Lett. B 55 (1975), 224-226.

Fradkin E.S., Vilkovisky G.A., Quantization of relativistic systems with constraints, CERN Preprint TH 2332-CERN, 1977.

[30] Gadella M., del Olmo M.A., Tosiek J., Geometrical origin of the *-product in the Fedosov formalism, J. Geom. Phys. 55 (2005), 316-352, hep-th/0405157.

[31] Gelfand I., Retakh V., Shubin M., Fedosov manifolds, Adv. Math. 136 (1998), 104-140, dg-ga/9707024.

[32] Geyer B., Lavrov P.M., Fedosov supermanifolds: Basic properties and the difference in even and odd cases, Internat. J. Modern Phys. A 19 (2004), 3195-3208, hep-th/0306218.

[33] Grigoriev M.A., Lyakhovich S.L., Fedosov deformation quantization as a BRST theory, Comm. Math. Phys. 218 (2001), 437-457, hep-th/0003114.

[34] Groenewold H.J., On the principles of elementary quantum mechanics, Physica 12 (1946), 405-460.

[35] Henneaux M., Teitelboim C., Quantization of gauge systems, Princeton University Press, Princeton, NJ, 1992.

[36] Karabegov A.V., Deformation quantizations with separation of variables on a Kähler manifold, Comm. Math. Phys. 180 (1996), 745-756, hep-th/9508013.

[37] Karabegov A.V., On the dequantization of Fedosov's deformation quantization, Lett. Math. Phys. 65 (2003), 133-146, math.QA/0307381.

[38] Karabegov A.V., Schlichenmaier M., Almost-Kähler deformation quantization, Lett. Math. Phys. 57 (2001), 135-148, math.QA/0102169.

[39] Kontsevich M., Deformation quantization of Poisson manifolds, Lett. Math. Phys. 66 (2003), 157-216, q-alg/9709040.

[40] Kravchenko O., How to calculate the Fedosov star-product (exercices de style), math.SG/0008157.

[41] Moyal J.E., Quantum mechanics as a statistical theory, Proc. Cambridge Philos. Soc. 45 (1949), 99-124. 
[42] Neumaier N., Universality of Fedosov's construction for star products of Wick type on pseudo-Kähler manifolds, Rep. Math. Phys. 52 (2003), 43-80.

[43] Xu P., Fedosov *-products and quantum momentum maps, Comm. Math. Phys. 197 (1998), 167-197, q-alg/9608006.

[44] Rothstein M., The structure of supersymplectic supermanifolds, in Differential Geometric Methods in Theoretical Physics (Rapallo, 1990), Lecture Notes in Phys., Vol. 375, Springer, Berlin, 1991, 331-343.

[45] Vaisman I., Fedosov quantization on symplectic ringed spaces, J. Math. Phys. 43 (2002), 283-298, math.SG/0106070.

[46] De Wilde M., Lecomte P.B.A., Existence of star-products and of formal deformations of the Poisson Lie algebra of arbitrary symplectic manifolds, Lett. Math. Phys. 7 (1983), 487-496. 